\title{
Potential mechanisms mediating sustained weight loss following Roux-en-Y gastric bypass and Sleeve Gastrectomy
}

\author{
J.M. Makaronidis ${ }^{1,2,3}$ and R.L. Batterham ${ }^{1,2,3}$
}

\section{Author Affiliations}

1 Centre for Obesity Research, Rayne Institute, Department of Medicine, University College London, London WC1E 6JF, United Kingdom.

2 University College London Hospitals (UCLH) Bariatric Centre for Weight Loss, Metabolic and Endocrine Surgery, UCLH, Ground Floor West Wing, 250 Euston Road, London NW1 2PG, United Kingdom.

3 National Institute of Health Research University College London Hospitals Biomedical Research Centre, London W1T 7DN, United Kingdom

\author{
†Correspondence to: \\ Prof Rachel Batterham \\ Centre for Obesity Research \\ University College London \\ Rayne Building \\ 5 University Street \\ London WC1E 6JF \\ UK
}

\section{Disclosure Statement: None}

\section{Key words}

Obesity

Bariatric surgery

Sleeve gastrectomy

Roux-en-Y gastric bypass

Gut hormones

Bile acids

Eating behavior

Microbiome

\section{Key Points}

1. Reduced energy intake, as a result of altered eating behavior, is the main driver for weight loss in humans following RYGB and SG.

2. The biological mediators underlying altered eating behavior post-surgery remain incompletely understood but changed gut-derived signals as a consequence of altered nutrient and/or biliary flow are key candidates.

3. Understanding the interplay between gut hormones, bile acids and the microbiome and their relative roles will enable the development of non-surgical treatment options for obesity.

\section{Synopsis}

Bariatric surgery is the only effective treatment for severe obesity. Roux-en-Y gastric bypass (RYGB) and sleeve gastrectomy (SG), the most commonly performed procedures, lead to sustained weight loss, improvements in obesity-related co-morbidities and reduced mortality. In humans the main driver for weight loss following RYGB and SG is reduced energy intake. Reduced appetite, changes in subjective taste and food preference and altered neural response to food cues are thought to drive altered eating behavior. The biological mediators underlying these changes remain incompletely understood but changes in gut-derived signals, as a consequence of altered nutrient and/or biliary flow, are key candidates. 


\section{Introduction}

Bariatric surgery is currently the only effective treatment for severe obesity, which is defined by a body mass index (BMI) equal to or greater than $40 \mathrm{~kg} / \mathrm{m}^{2}$, or greater than $35 \mathrm{~kg} / \mathrm{m}^{2}$ in the presence of obesity-related complications (1). Bariatric surgery involves surgical manipulation of the gastrointestinal (GI) tract, which alters nutrient flow and impacts upon Gl biology. These changes engender beneficial effects upon energy and glucose homeostasis (2-4).

Gaining an understanding of the mechanisms underlying the sustained weight loss and metabolic benefits produced by bariatric surgery may hold the key to developing novel non-surgical treatments for obesity and type 2 diabetes (T2D). In particular, it is now clear that altered eating behavior plays a key role in mediating the weight loss following SG and RYGB in humans $(5,6)$. This has led to intense research efforts focused on delineating how SG and RYGB impact upon the drive to eat.

\section{Historical perspectives}

The concept of bariatric surgery emerged in the 1950s from the observation that small intestine resection resulted in weight loss (7). The first procedure aimed specifically at inducing weight loss, a jejunoileal bypass (JIB), where the majority of the small intestine was bypassed, was performed in 1953 (8). The theoretical basis driving the development of the JIB was that weight loss would be achieved through malabsorption. This indeed was the case, however weight loss was coupled with electrolyte imbalances, nutritional deficiencies, diarrhea and liver failure, necessitating the development of less malabsorptive surgical procedures (8). In addition, patients with peptic ulcer disease who underwent gastric resection and/or bypass were also observed to lose weight. In these patients reduced stomach capacity (restriction) and decreased digestion were suggested to drive weight loss (8). This led Mason et al. to perform the first gastric bypass procedure for weight loss in 1967 (8). These two different approaches formed the basis for subsequent "malabsorptive" and "restrictive" procedures or "hybrid" procedures, combining these two mechanisms, for example the RYGB, that have evolved over the ensuing 60 years $(7,8)$. However, it is now recognized that the majority of bariatric procedures engender weight loss and metabolic improvements by mechanisms other than restriction and/or malabsorption. 
Over the last decade the beneficial effects of bariatric surgery upon weight and obesityassociated co-morbidities have resulted in a marked increase in the number of bariatric procedures undertaken with approximately 460,000 operations performed in 2013 (9). The choice of surgical procedure has, and still is, evolving guided by technical advances, the beneficial clinical outcomes achieved, short and long-term complication rates as well as by the evidence regarding the physiology underpinning their success. Currently, the most common procedures undertaken globally are RYGB, SG, adjustable gastric banding ( $A B G$ ) and biliopancreatic duodenal switch (9). Purely restrictive procedures such as AGB and vertical banded gastroplasty are now less commonly performed. RYGB and SG represent the vast majority of procedures undertaken and will form the focus of this review.

Figure 1 near here

\section{RYGB}

In RYGB, a small gastric pouch of approximately $20 \mathrm{mls}$ is created through dividing the stomach. This is anastomosed with the mid-jejunum, creating the Roux limb, allowing ingested nutrients to bypass the majority of the stomach, duodenum and proximal jejunum (10). Anastomosis of the biliopancreatic limb with the jejunum allows drainage of bile acids and pancreatic secretions, which mix with the ingested nutrients in the jejunum (10). RYGB is established as an efficient treatment for severe obesity, with the long-term (>20 years) outcome data demonstrating sustained weight loss and favorable metabolic outcomes, particularly relating to T2D $(11,12)$.

SG

SG, intended as a purely restrictive procedure, was initially performed as a first-stage procedure to reduce weight in patients with $\mathrm{BMl}>50 \mathrm{~kg} / \mathrm{m}^{2}$. Following weight loss, this was then transformed into a hybrid procedure such as RYGB. However, SG alone resulted in significant sustained weight loss and metabolic benefits, leading to the adoption of $S G$ as a standalone procedure $(9,13,14)$. SG involves transection along the greater curvature of the stomach and removing $80-90 \%$ of the total stomach volume, including the fundus and body, without intervention to the small intestine (15). Gastric contents pass rapidly into the duodenum.

SG is technically easier than RYGB, associated with fewer complications, peri-operative and nutritional complications and produces similar short-term weight-loss and clinical outcomes compared 
to RYGB $(13,16,17)$. Consequently the proportion of SG has increased from 0 to $37 \%$ from $2003-$ 2013 and is anticipated to become the most common bariatric procedure undertaken (9). Randomized control trials are currently underway, comparing outcomes post-RYBG against SG in severely obese individuals and comparing the efficacy of the two procedures with regards to resolution of T2D (18, 19).

\section{FIGURE 2 near here}

Table 1 near here

\section{Body weight regulation and the impact of obesity}

The mechanisms regulating body weight are complex and involve neural circuits controlling energy homeostasis as well as reward-related pathways. Regulatory brain centers continually integrate peripheral signals relating to energy stores and nutrient availability to determine feeding behavior (4). Leptin, insulin and gut-derived factors are established as key regulators of energy homeostasis (20). Peripheral long-term signals, providing information regarding availability of energy stores via insulin and leptin secretion in response to adiposity, and short-term signals regarding nutrient and meal-derived energy availability modulate hunger and satiety perception (21).

Gut hormones are secreted from the Gl enteroendocrine cells in response to nutrient ingestion and act as autocrine, paracrine and endocrine regulators of energy and glucose homeostasis $(4,22)$. The anorectic hormone peptide YY3-36 (PYY) and glucagon-like peptide-1 (GLP-1), an incretin hormone, are both secreted in response to nutrient ingestion from enteroendocrine L-cells present throughout the Gl tract $(5,22)$. PYY has potent anorectic effects, with exogenous administration shown to reduce food intake (23). Evidence from experimental imaging and translational studies indicate that PYY mediates its anorectic effects predominantly by acting upon central appetiteregulating circuits and brain regions involved in food reward (24). There is also evidence that circulating GLP-1 has appetite-suppressing effects by modulating neural activity within homeostatic and reward brain centers, in a manner additive to PYY (25). The orexigenic hormone ghrelin, produced by P/D1 cells in oxyntic glands in the gastric fundus, also acts on homeostatic and reward centers involved in appetite regulation to control energy intake (26). Circulating ghrelin levels increase in the fasted state and decrease post-prandially $(22,26)$. Elevations in ghrelin levels have been shown to enhance the hedonic responses to eating (27). 
Bile acids, in addition to their role in lipid metabolism, influence glucose and energy homeostasis, through signaling and nutrient sensing in the hepatoportal circulation (28). Furthermore, bile acids directly affect the intestinal microbiome through local effects on the intestinal milieu (29). The microbiome, which has recently been implicated in the pathophysiology of several disease processes, also contributes toward energy homeostasis $(28,30)$. A dysbiotic relationship between host and micriobiome could contribute to the development of obesity through increasing intestinal permeability and energy absorption (28).

Taste and olfactory signals influence food selection and, consequently, energy intake (31) . Interestingly, there is emerging evidence for an interplay between signals of energy homeostasis and taste and smell. Insulin leptin, GLP-1, PYY and ghrelin have been found in saliva and their cognate receptors identified on taste buds and olfactory neurons (31). It is now also clear that rewarding foodrelated sensory stimuli can override satiety signals leading to excess energy intake. Chronic excess energy intake leads to deregulation of the homeostatic mechanisms that normally control body weight, which will predispose an obese individual to further weight gain. (27). Physiological changes in obesity include: resistance to the effects of both leptin and insulin, blunted circulating gut hormone responses to nutrient ingestion, with reduced plasma PYY and GLP-1 levels, dysregulation of circulating ghrelin, reduced circulating bile acids levels, altered gut microbiome and decreased vagal signal transmission (32).

Obese individuals perceive foods high in sugar and fat content more pleasurable compared to lean individuals (33). Furthermore, brain imaging studies have shown increased stimulation of central reward pathways in response to eating or food cues in obese compared to normal weight individuals (33-35). Consequently, eating behavior in obesity becomes dissociated form perceptions of satiety and hunger (36).

\section{Why is weight loss maintenance through non-surgical means so difficult?}

Weight loss through intentional caloric restriction results in compensatory changes that aim to defend the higher body weight (35). These include decreased energy expenditure, due to reduced of lean muscle mass and reduced sympathetic activity (37), reduced circulating leptin, GLP-1 and PYY levels with increased ghrelin levels (35), altered brain neural response to food cues. Moreover, these 
changes are sustained in the long term (35). Table 2 compares physiological responses to weight loss though dieting with bariatric surgery.

Table 2 near here

\section{Sustained weight loss following bariatric surgery}

In contrast to weight loss though calorie-restricted dieting, bariatric surgery poses an effective treatment for severe obesity with significant weight loss, sustained in the long-term. Data from the Swedish Obese Subjects (SOS) study shows that after 20 years of follow up, patients who received bariatric surgery had achieved a mean body weight reduction of $18 \%$ compared to a $1 \%$ mean body weight reduction seen in patients who received standard medical treatment through their local health centres. (11). However, it is important to note that the weight loss achieved post-surgery follows a wide and normal distribution and studying the extreme responders may provide key insights into the mechanisms involved (Figure 3) (38).

\section{FIGURE 3 near here}

\section{Mechanisms other than restriction and malabsorption are at play}

The multi-factorial mechanisms promoting weight loss and improvements in obesity-related comorbidities following RYGB/SG are incompletely understood. However, it is now clear that the beneficial effects of bariatric surgery are not achieved through restriction and malabsorption alone (39, 40). Reduced energy intake, as a result of altered eating behavior, is the main driver for weight loss in humans $(5,22,41)$. Here we review the key eating behavior changes that occur following RYGB and SG together with the potential mechanisms by which RYGB and SG impact upon central appetiteregulating circuits.

\section{Eating behavior changes following RYGB and SG}

Changes in eating behavior following bariatric surgery were first suggested by Bray et al (42) in 1980 , who observed a reduction in food intake following intestinal bypass. They proposed that 'oral' (taste or smell), 'gastrointestinal' (mechanical distension or nutrient composition) and 'postingestional' (nutrient or hormonal satiety mediators) factors as possible mediators (42). Following RYGB and SG patients report appetite changes with reduced hunger and increased satiety (43-45). Post-surgery altered subjective taste and smell together with the food aversions are common and are 
thought to drive a change in food preferences away from sweet and high-fat foods $(6,46,47)$. Moreover, the reward value of food-related stimuli is decreased post-surgery $(6,48)$. A study using progressive ratio tasks to assess reward values of food pre- and post-RYGB, revealed a significant reduction in the reward value of sweet and high-fat foods after surgery, compared to a control group (49). Studies using functional MRI scanning have shown lower brain-hedonic responses to food following RYGB compared to AGB, associated with lower palatability of high-fat and energy-dense foods $(48,50,51)$. Consequently, a change in the way food is perceived is considered a key contributor toward the altered food preference leading to a lower energy intake post-surgery.

\section{Potential mechanisms underlying eating behavior changes post-surgery in humans}

Altered eating behavior following RYGB and SG results in a lower energy intake, which is the main driver for weight loss in humans $(22,52)$. The biological mediators underlying these changes remain incompletely understood but gut-derived signals as a consequence of altered nutrient and/or biliary flow are key candidates. Interestingly, although RYGB and SG differentially alter Gl anatomy, they lead to comparable outcomes in terms of weight loss and metabolic benefits $(17,53)$.

\section{Gut hormones}

A landmark publication by Cummings et al. in 2002, showed that circulating ghrelin levels rose with calorie-restricted diets but reduced markedly post-RYGB (54). These findings acted as the catalyst for investigating the role of gut hormones as mediators of the beneficial effects of surgery. Subsequently, there has been some controversy regarding post-RYGB circulating ghrelin levels. However, these differences reflect methodological variability in terms of duration post-surgery and sample processing techniques as the active from of ghrelin, acyl-ghrelin is highly labile and requires specific processing in order to accurately measure circulating levels $(55,56)$. SG, which involves removing the majority of ghrelin-producing cells, leads to sustained and greater reduction in circulating acyl-ghrelin levels than RYGB (53). Both RYGB and SG are associated with marked increase in nutrient-stimulated circulating levels of PYY and GLP-1 $(53,57)$. These changes occur prior to and independent of weight loss, pointing towards a procedure-related mechanism. Comparative studies suggest that RYGB leads to greater post-meal PYY and GLP-1 levels compared to $S G(53)$. 
Cross-sectional studies have shown that patients with poor weight loss exhibit higher subjective hunger, lower subjective satiety, higher ghrelin and lower PYY and GLP-1 compared to patients with good weight loss $(58,59)$. Furthermore, inhibition of PYY and GLP-1 with somatostatin analogue, octreotide, leads to return of appetite and increased energy intake and weight gain (60).

Gut hormone receptors for PYY, GLP-1 and ghrelin are expressed on taste buds and there is emerging evidence that these hormones modulate taste $(61,62)$. Given that the post-operative changes in eating behavior and taste perception overlap with the known effects of changes in gut hormones, it is biologically plausible that these changes can be attributed to increased gut hormone secretion and signaling through gut hormones receptors. Favorable gut hormone responses postsurgery, with increased meal-stimulated PYY and GLP-1 levels, combined with lower ghrelin levels, are clearly associated with higher weight loss. However, the causative factor of this effect is not yet established.

RYGB and SG have been undertaken in transgenic rodents in order to provide mechanistic insights into the role of altered gut hormones. Somewhat surprisingly, global GLP-1 receptor knockout mice exhibit similar weight loss and glycemic improvement following SG and RYGB to their wild-type littermates $(63,64)$. Similarly, global ghrelin knockout mice respond in a similar manner following SG to their wild-type control mice (65). However, PYY knockout mice exhibit reduced early post-operative weight loss and glycemic improvement compared to wild-type mice $(4,65)$. These observations suggest that weight loss and metabolic improvements are at least partially mediated by post-operative modulation of PYY.

Other gut hormones with effects on feeding behavior have also been studied. Oxyntomodulin, a pro-glucagon derived peptide with parallel actions to GLP-1 and anorectic effects, has been shown to increase post-RYGB (22). The anorexigenic hormone cholecystokinin has been suggested to act synergistically with leptin and amylin, a pancreatic hormone co-secreted with insulin, may also exhibit anorectic effects (20). However, the extent of their role in mediating the effects of post-bariatric surgery has not been established.

\section{Bile acids and microbiome}


Both RYGB and SG induce changes in the interaction between ingested nutrients and bile acids. Despite their anatomical differences, they exert similar effects on bile acids, altering both their composition and secretion (29). RYGB and SG both lead to increased bile acid secretion, which has been linked to improved lipid and glucose metabolism (28).

The metabolic effects of bile acids are mediated through signaling via two receptors; the cell surface G protein-coupled receptor 5 (TGR5) and farsenoid X receptor (FXR) (29). TGR5 is expressed on L-cells and activation of the receptor leads to GLP-1 release. Increased TGR5 activation is a proposed mechanism contributing to higher GLP-1 levels following bariatric surgery (28). The FXR pathways have several functions, including regulating bile acid synthesis, secretion, conjugation and regulation of the intestinal microbiome $(28,29,66,67)$. The latter influences energy absorption, through altering intestinal mucosal permeability, and energy expenditure by intracellular thyroid hormone activation via FXR signalling $(28,68)$. Following RYGB and SG the intestinal microbiome is altered. Moreover, the finding that transplant of feces from RYGB-treated to germ-free mice resulted in significant greater weight loss compared to mice receiving feces from sham-surgery treated mice suggests that the altered microbiome per se contributes to weight loss (69). However, significant differences exist between the rodent and the human microbiome, as well as in the physiological processes driving weight loss. Thus, the relationship between post-operative weight loss and bile acids, FXR signaling and intestinal bacteria in humans remains to be clarified.

\section{Enteroplasticity}

Enteroplasticity refers to the post-surgical adaptations, including remodeling of the intestinal mucosa, morphological changes and alterations in innervation (29). Increased post-operative nutrient exposure of intestinal L-cells is thought to lead to enhanced secretion of PYY, GLP-1 and oxyntomodulin $(28,29)$. There is also evidence that L-cells proliferate and exhibit increased nutrient sensitivity, exhibiting higher responsiveness to the exposed nutrient with resulting higher nutrientstimulated PYY and GLP-1 secretion (29). Neurophysiological studies suggest that vagus nerve signaling also increases post-RYGB (32). These intestinal adaptations may contribute to the sustained metabolic effects of bariatric surgery.

\section{The future: knife-less surgery?}


Bariatric surgery is a safe and highly effective treatment for obesity and T2D. Nevertheless, it requires long-term follow-up and monitoring for complications, nutritional deficiencies and development or relapse of T2D. Despite the increasing numbers of procedures performed, there is high geographic variability in accessibility to surgery. In addition, although bariatric surgery is highly effective, at the individual level clinical response is highly variable. Therefore, developing novel therapies that mimic the post-bariatric surgery internal milieu poses an appealing therapeutic aim. Understanding the interplay between gut hormones, bile acids and the microbiome will be key in order to both fully elucidate both the success of bariatric surgery as well as the reasons behind the variability in response. Such findings would enable the development of non-surgical treatment options for obesity, leading to sustainable weight loss and reduction in obesity-related morbidity and mortality. 


\section{Figure legends}

Figure 1: Left: Number of bariatric procedures performed by published surveys. Right: Numbers of procedures performed worldwide. Adapted from Angrisani et al (9)

Figure 2: Illustrations of (A) normal GI tract, (B) RYGB and (C) SG.

(A) Multiple hormones are secreted from the GI tract. Ghrelin is secreted from the P/D1 cells of the gastric fundus, whereas GLP-1 and PYY are secreted from L-cells located predominantly in the ileum and colon. (B) RYGB; nutrients bypass the majority of the stomach and flow directly into the midjejunum. Anastomosis of the biliopancreatic limb with the jejunum allows drainage of bile acids into the common limb, where ingested nutrients and bile acids mix. (C) SG; Nutrients pass rapidly from the gastric sleeve into the duodenum with unaltered flow of bile acids. Following RYGB and SG, circulating ghrelin levels are reduced, meal-stimulated PYY and GLP-1 are increased. Bile acid secretion is enhanced and the microbiome is altered. These changes in gut-derived factors are thought to drive reduced appetite and altered food reward value, leading to a decreased energy intake and weight loss.

Figure 3: Histogram illustrating maximal percent weight loss in patients following RYGB and SG. Adapted from Manning et al (38) 


\section{$\underline{\text { Tables }}$}

Table 1: Weight loss, type 2 diabetes remission and complications following RYGB and SG

\begin{tabular}{|ccc|} 
& RYGB & SG \\
\hline $\begin{array}{l}\text { Weight loss (\%WL) } \\
\text { STAMPEDE (3 years)(70) }\end{array}$ & $24.5+/-9.1 \%$ & $21.1+/-8.9 \%$ \\
BOLD data 6 months (13) & $26.8 \%$ & $24 \%$ \\
BOLD data 12 months(13) & $34.2 \%$ & $29.5 \%$ \\
Type 2 diabetes remission at 3 years & & \\
STAMPEDE(70) & $38 \%$ & $24 \%$ \\
Complications (BOLD data) (13) & & \\
All complications & & \\
Serious complications & $10 \%$ & $6.3 \%(P<0.001)$ \\
\end{tabular}

The Surgical TreAtment and Medications Potentially Eradicate Diabetes Efficiently (70) trial, investigated 3-year weight loss and remission of type 2 diabetes in patients with type 2 diabetes, randomised to medical therapy or medical therapy plus either RYGB or SG (16). The Bariatric Outcomes Longitudinal Database (BOLD) compared weight loss and complications following SG and RYGB (13). 
Table 2: Comparison of the effects of weight loss due to 'dieting' or following bariatric surgery on signals of energy balance and appetite.

\begin{tabular}{|c|c|c|}
\hline & Weight loss through dieting & $\begin{array}{l}\text { Weight loss through } \\
\text { bariatric surgery }\end{array}$ \\
\hline Ghrelin & $\begin{array}{l}\text { Levels increase with dieting ( } 35 \text {, } \\
54 \text { ) }\end{array}$ & $\begin{array}{l}\text { Reduction with SG, possible } \\
\text { reduction with RYGB in the } \\
\text { immediate post-operative } \\
\text { period(53) }\end{array}$ \\
\hline PYY & Reduced levels $(28,35)$ & Increased levels $(17,53,72)$ \\
\hline GLP-1 & Reduced levels $(28,35)$ & Increased levels $(17,22,53)$ \\
\hline Leptin & Reduced levels(71) & $\begin{array}{l}\text { Reduced levels.(71) } \\
\text { Suggestion that leptin } \\
\text { sensitivity may improve (20) }\end{array}$ \\
\hline Bile acids & $\begin{array}{l}\text { Unclear. } \\
\text { Blunted bile acid response in } \\
\text { obesity with no significant } \\
\text { difference following AGB (73) }\end{array}$ & Increased secretion \\
\hline Intestinal microbiome & Improvement with weight loss (74) & $\begin{array}{l}\text { Altered ('leaner') microbiome } \\
(29,74)\end{array}$ \\
\hline Perceived hunger & Increased hunger (35) & Reduced hunger (43) \\
\hline Perceived satiety & Lower satiety (35) & Increased satiety (6) \\
\hline Food aversions & & Altered food preference (47) \\
\hline $\begin{array}{l}\text { Long-term } \\
\text { sustainability }\end{array}$ & $\begin{array}{l}\text { Average } 80 \% \text { weight regain in } 5 \\
\text { years (75) }\end{array}$ & $\begin{array}{l}\text { Average weight loss of } 25 \% \\
\text { at } 10 \text { and } 18 \% \text { at } 20 \text { years } \\
\text { post RYGB }(3,11)\end{array}$ \\
\hline $\begin{array}{l}\text { Body weight } \\
\text { regulation }\end{array}$ & $\begin{array}{l}\text { Homeostatic mechanisms defend } \\
\text { higher body weight }(35,75)\end{array}$ & $\begin{array}{l}\text { Resets body weight 'set } \\
\text { point' to lower weight (5) }\end{array}$ \\
\hline
\end{tabular}




\section{References}

*1. Gloy VL, Briel M, Bhatt DL, et al. Bariatric surgery versus non-surgical treatment for obesity: a systematic review and metaanalysis of randomised controlled trials. Bmj. 2013;347:f5934.

*2. Buchwald H, Avidor Y, Braunwald E, et al. Bariatric surgery: a systematic review and meta-analysis. Jama. 2004;292(14):1724-

37.

*3. Buchwald H, Estok R, Fahrbach K, et al. Weight and type 2 diabetes after bariatric surgery: systematic review and meta-analysis.

The American journal of medicine. 2009;122(3):248-56.e5.

4. Miras AD, le Roux CW. Mechanisms underlying weight loss after bariatric surgery. Nature reviews Gastroenterology \& hepatology. 2013;10(10):575-84.

5. Manning S, Pucci A, Batterham RL. Roux-en-Y gastric bypass: effects on feeding behavior and underlying mechanisms. The Journal of clinical investigation. 2015;125(3):939-48.

6. le Roux CW, Bueter M. The physiology of altered eating behaviour after Roux-en-Y gastric bypass. Experimental physiology. 2014;99(9):1128-32.

7. Saber AA, Elgamal MH, McLeod MK. Bariatric surgery: the past, present, and future. Obes Surg. 2008;18(1):121-8.

8. Buchwald H. The evolution of metabolic/bariatric surgery. Obes Surg. 2014;24(8):1126-35.

9. Angrisani L, Santonicola A, Iovino P, et al. Bariatric Surgery Worldwide 2013. Obes Surg. 2015;25(10):1822-32.

10. Olbers T, Lonroth H, Fagevik-Olsen M, et al. Laparoscopic gastric bypass: development of technique, respiratory function, and long-term outcome. Obes Surg. 2003;13(3):364-70.

11. Sjostrom L. Review of the key results from the Swedish Obese Subjects (SOS) trial - a prospective controlled intervention study of bariatric surgery. Journal of internal medicine. 2013;273(3):219-34.

12. Sjostrom L, Peltonen M, Jacobson P, et al. Association of bariatric surgery with long-term remission of type 2 diabetes and with microvascular and macrovascular complications. Jama. 2014;311(22):2297-304.

13. Sczepaniak JP, Owens ML, Shukla H, et al. Comparability of weight loss reporting after gastric bypass and sleeve gastrectomy using BOLD data 2008-2011. Obes Surg. 2015;25(5):788-95.

14. Carlin AM, Zeni TM, English WJ, et al. The comparative effectiveness of sleeve gastrectomy, gastric bypass, and adjustable gastric banding procedures for the treatment of morbid obesity. Annals of surgery. 2013;257(5):791-7.

15. Abu-Jaish W, Rosenthal RJ. Sleeve gastrectomy: a new surgical approach for morbid obesity. Expert review of gastroenterology \& hepatology. 2010;4(1):101-19.

16. Schauer PR, Bhatt DL, Kirwan JP, et al. Bariatric surgery versus intensive medical therapy for diabetes--3-year outcomes. N Engl J Med. 2014;370(21):2002-13.

17. Peterli R, Steinert RE, Woelnerhanssen B, et al. Metabolic and hormonal changes after laparoscopic Roux-en-Y gastric bypass and sleeve gastrectomy: a randomized, prospective trial. Obes Surg. 2012;22(5):740-8.

18. Göteborg University. Sleeve Gastrectomy and Roux-en-Y Gastric Bypass in the Treatment of Type 2 Diabetes Mellitus ClinicalTrials.gov [Internet]: Bethesda (MD): National Library of Medicine (US) NCT Number: NCT01984762; 2015 [cited 2105 Nov 20]. Available from: https://clinicaltrials.gov/ct2/show/NCT01984762?term=Sleeve+Gastrectomy+and+Roux-en-

$\underline{Y}+$ Gastric+Bypass+in+the+Treatment+of+Type+2+Diabetes+Mellitus\&rank=1. 
National Library of Medicine (US) NCT Number: NCT02475590; 2015. Available from:

https://clinicaltrials.gov/ct2/show/record/NCT02475590?term=obesity+sleeve+gastrectomy+roux-en-y\&rank=3.

20. Suzuki K, Jayasena CN, Bloom SR. Obesity and appetite control. Experimental diabetes research. 2012;2012:824305.

21. Morton GJ, Meek TH, Schwartz MW. Neurobiology of food intake in health and disease. Nature reviews Neuroscience. 2014;15(6):367-78.

22. Scott WR, Batterham RL. Roux-en-Y gastric bypass and laparoscopic sleeve gastrectomy: understanding weight loss and improvements in type 2 diabetes after bariatric surgery. Am J Physiol Regul Integr Comp Physiol. 2011;301(1):R15-27.

23. Batterham RL, Cowley MA, Small CJ, et al. Gut hormone PYY(3-36) physiologically inhibits food intake. Nature. 2002;418(6898):650-4.

24. Batterham RL, ffytche DH, Rosenthal JM, et al. PYY modulation of cortical and hypothalamic brain areas predicts feeding behaviour in humans. Nature. 2007;450(7166):106-9.

25. Manning S, Pucci A, Batterham RL. GLP-1: a mediator of the beneficial metabolic effects of bariatric surgery? Physiology (Bethesda, Md). 2015;30(1):50-62.

26. Muller TD, Nogueiras R, Andermann ML, et al. Ghrelin. Molecular metabolism. 2015;4(6):437-60.

27. Berthoud HR. Metabolic and hedonic drives in the neural control of appetite: who is the boss? Current opinion in neurobiology. 2011;21(6):888-96.

28. Dixon JB, Lambert EA, Lambert GW. Neuroendocrine adaptations to bariatric surgery. Molecular and cellular endocrinology. 2015.

29. Seeley RJ, Chambers AP, Sandoval DA. The role of gut adaptation in the potent effects of multiple bariatric surgeries on obesity and diabetes. Cell Metab. 2015;21(3):369-78.

30. Aron-Wisnewsky J, Clement K. The effects of gastrointestinal surgery on gut microbiota: potential contribution to improved insulin sensitivity. Curr Atheroscler Rep. 2014;16(11):454.

31. Cummings DE. Taste and the regulation of food intake: it's not just about flavor. The American journal of clinical nutrition. 2015;102(4):717-8.

32. Browning KN, Fortna SR, Hajnal A. Roux-en-Y gastric bypass reverses the effects of diet-induced obesity to inhibit the responsiveness of central vagal motoneurones. The Journal of physiology. 2013;591(Pt 9):2357-72.

33. Rissanen A, Hakala P, Lissner L, et al. Acquired preference especially for dietary fat and obesity: a study of weight-discordant monozygotic twin pairs. International journal of obesity and related metabolic disorders : journal of the International Association for the Study of Obesity. 2002;26(7):973-7.

34. Shin AC, Townsend RL, Patterson LM, et al. "Liking" and "wanting" of sweet and oily food stimuli as affected by high-fat dietinduced obesity, weight loss, leptin, and genetic predisposition. Am J Physiol Regul Integr Comp Physiol. 2011;301(5):R1267-80.

35. Sumithran P, Prendergast LA, Delbridge E, et al. Long-term persistence of hormonal adaptations to weight loss. N Engl J Med. 2011;365(17):1597-604.

36. Barkeling B, King NA, Naslund E, et al. Characterization of obese individuals who claim to detect no relationship between their eating pattern and sensations of hunger or fullness. Int J Obes (Lond). 2007;31(3):435-9.

37. Leibel RL, Hirsch J. Diminished energy requirements in reduced-obese patients. Metabolism: clinical and experimental. 1984;33(2):164-70. 
Roux-en-Y gastric bypass. Surgical endoscopy. 2015;29(6):1484-91.

39. Dirksen C, Damgaard M, Bojsen-Moller KN, et al. Fast pouch emptying, delayed small intestinal transit, and exaggerated gut hormone responses after Roux-en-Y gastric bypass. Neurogastroenterology and motility : the official journal of the European Gastrointestinal Motility Society. 2013;25(4):346-e255.

40. Odstrcil EA, Martinez JG, Santa Ana CA, et al. The contribution of malabsorption to the reduction in net energy absorption after long-limb Roux-en-Y gastric bypass. The American journal of clinical nutrition. 2010;92(4):704-13.

41. Lutz TA, Bueter M. The physiology underlying Roux-en-Y gastric bypass: a status report. Am J Physiol Regul Integr Comp Physiol. 2014;307(11):R1275-91.

42. Bray GA, Dahms WT, Atkinson RL, et al. Factors controlling food intake: a comparison of dieting and intestinal bypass. The American journal of clinical nutrition. 1980;33(2 Suppl):376-82.

43. Warde-Kamar J, Rogers M, Flancbaum L, et al. Calorie intake and meal patterns up to 4 years after Roux-en-Y gastric bypass surgery. Obes Surg. 2004;14(8):1070-9.

44. Mathes CM, Spector AC. Food selection and taste changes in humans after Roux-en-Y gastric bypass surgery: a direct-measures approach. Physiology \& behavior. 2012;107(4):476-83.

45. Laurenius A, Larsson I, Bueter M, et al. Changes in eating behaviour and meal pattern following Roux-en-Y gastric bypass. Int J Obes (Lond). 2012;36(3):348-55.

46. Thomas JR, Marcus E. High and low fat food selection with reported frequency intolerance following Roux-en-Y gastric bypass. Obes Surg. 2008;18(3):282-7.

47. Holinski F, Menenakos C, Haber G, et al. Olfactory and Gustatory Function After Bariatric Surgery. Obes Surg. 2015.

48. Scholtz S, Miras AD, Chhina N, et al. Obese patients after gastric bypass surgery have lower brain-hedonic responses to food than after gastric banding. Gut. 2014;63(6):891-902.

49. Miras AD, Jackson RN, Jackson SN, et al. Gastric bypass surgery for obesity decreases the reward value of a sweet-fat stimulus as assessed in a progressive ratio task. The American journal of clinical nutrition. 2012;96(3):467-73.

50. Ochner CN, Kwok Y, Conceicao E, et al. Selective reduction in neural responses to high calorie foods following gastric bypass surgery. Annals of surgery. 2011;253(3):502-7.

51. Ochner CN, Stice E, Hutchins E, et al. Relation between changes in neural responsivity and reductions in desire to eat highcalorie foods following gastric bypass surgery. Neuroscience. 2012;209:128-35.

52. Shin AC, Berthoud HR. Obesity surgery: happy with less or eternally hungry? Trends in endocrinology and metabolism: TEM. $2013 ; 24(2): 101-8$

53. Yousseif A, Emmanuel J, Karra E, et al. Differential effects of laparoscopic sleeve gastrectomy and laparoscopic gastric bypass on appetite, circulating acyl-ghrelin, peptide YY3-36 and active GLP-1 levels in non-diabetic humans. Obes Surg. 2014;24(2):241-52.

54. Cummings DE, Weigle DS, Frayo RS, et al. Plasma ghrelin levels after diet-induced weight loss or gastric bypass surgery. N Engl J Med. 2002;346(21):1623-30.

55. Chandarana K, Drew ME, Emmanuel J, et al. Subject standardization, acclimatization, and sample processing affect gut hormone levels and appetite in humans. Gastroenterology. 2009;136(7):2115-26.

56. Hosoda H, Kangawa K. Standard sample collections for blood ghrelin measurements. Methods in enzymology. 2012;514:113-26. 

prospective randomised trial. Journal of gastrointestinal surgery : official journal of the Society for Surgery of the Alimentary Tract. $2012 ; 16(6): 1116-22$

58. Dirksen C, Jorgensen NB, Bojsen-Moller KN, et al. Gut hormones, early dumping and resting energy expenditure in patients with good and poor weight loss response after Roux-en-Y gastric bypass. Int J Obes (Lond). 2013;37(11):1452-9.

59. le Roux CW, Welbourn R, Werling M, et al. Gut hormones as mediators of appetite and weight loss after Roux-en-Y gastric bypass. Annals of surgery. 2007;246(5):780-5.

60. Pucci A, Wui Hang C, Jenny J, et al. A case of severe anorexia, excessive weight loss and high peptide YY levels after sleeve gastrectomy. 2015 .

61. Mathes CM, Bueter M, Smith KR, et al. Roux-en-Y gastric bypass in rats increases sucrose taste-related motivated behavior independent of pharmacological GLP-1-receptor modulation. Am J Physiol Regul Integr Comp Physiol. 2012;302(6):R751-67.

62. Miras AD, le Roux CW. Bariatric surgery and taste: novel mechanisms of weight loss. Current opinion in gastroenterology. $2010 ; 26(2): 140-5$

63. Mokadem M, Zechner JF, Margolskee RF, et al. Effects of Roux-en-Y gastric bypass on energy and glucose homeostasis are preserved in two mouse models of functional glucagon-like peptide-1 deficiency. Molecular metabolism. 2014;3(2):191-201.

64. Ye J, Hao Z, Mumphrey MB, et al. GLP-1 receptor signaling is not required for reduced body weight after RYGB in rodents. Am J Physiol Regul Integr Comp Physiol. 2014;306(5):R352-62.

65. Chandarana K, Gelegen C, Karra E, et al. Diet and gastrointestinal bypass-induced weight loss: the roles of ghrelin and peptide YY. Diabetes. 2011;60(3):810-8.

66. Dutia R, Embrey M, O'Brien S, et al. Temporal changes in bile acid levels and 12alpha-hydroxylation after Roux-en-Y gastric bypass surgery in type 2 diabetes. Int J Obes (Lond). 2015;39(5):806-13.

67. Kuipers F, Groen AK. FXR: the key to benefits in bariatric surgery? Nat Med. 2014;20(4):337-8.

68. Watanabe M, Houten SM, Mataki C, et al. Bile acids induce energy expenditure by promoting intracellular thyroid hormone activation. Nature. 2006;439(7075):484-9.

69. Tremaroli V, Karlsson F, Werling M, et al. Roux-en-Y Gastric Bypass and Vertical Banded Gastroplasty Induce Long-Term Changes on the Human Gut Microbiome Contributing to Fat Mass Regulation. Cell Metab. 2015;22(2):228-38.

70. Schauer PR, Bhatt DL, Kirwan JP, et al. Bariatric surgery versus intensive medical therapy for diabetes--3-year outcomes. N Engl J Med. 2014;370(21):2002-13.

71. Sweeney TE, Morton JM. Metabolic surgery: action via hormonal milieu changes, changes in bile acids or gut microbiota? A summary of the literature. Best practice \& research Clinical gastroenterology. 2014;28(4):727-40.

72. le Roux CW, Aylwin SJ, Batterham RL, et al. Gut hormone profiles following bariatric surgery favor an anorectic state, facilitate weight loss, and improve metabolic parameters. Annals of surgery. 2006;243(1):108-14.

73. Pournaras DJ, Glicksman C, Vincent RP, et al. The role of bile after Roux-en-Y gastric bypass in promoting weight loss and improving glycaemic control. Endocrinology. 2012;153(8):3613-9.

74. Rosenbaum M, Knight R, Leibel RL. The gut microbiota in human energy homeostasis and obesity. Trends in endocrinology and metabolism: TEM. 2015;26(9):493-501.

75. Leibel RL, Seeley RJ, Darsow T, et al. Biologic Responses to Weight Loss and Weight Regain: Report From an American Diabetes Association Research Symposium. Diabetes. 2015;64(7):2299-309. 
